\title{
Photocontrolled Variations in the Wetting Capability of Photochromic Polymers Enhanced by Surface Nanostructuring
}

\author{
Athanassia Athanassiou, ${ }^{*, \dagger}$ Maria I. Lygeraki, ${ }^{\dagger,}$ Dario Pisignano, ${ }^{\S}$ Kleanthi Lakiotaki,${ }^{\dagger}$ \\ Maria Varda, ${ }^{\dagger, \|}$ Elisa Mele, ${ }^{\S}$ Costas Fotakis, ${ }^{\dagger}, \| l$ Roberto Cingolani, ${ }^{\S}$ and \\ Spiros H. Anastasiadis $*, \dagger, \|$
}

\begin{abstract}
Institute of Electronic Structure and Laser (IESL), Foundation for Research and Technology-Hellas (FORTH), 71110 Heraklion, Crete, Greece, Physics Department, University of Patras, 22500 Rio, Patras, Greece, National Nanotechnology Laboratory (NNL) of CNR, Distretto Technologico ISUFI, Via Arnesano, I-73100 Lecce, Italy, and Physics Department, University of Crete, 71003 Heraklio, Crete, Greece
\end{abstract}

Received August 3, 2005. In Final Form: December 21, 2005

\begin{abstract}
The wetting characteristics of surfaces of polymers doped with photochromic spiropyran molecules can be tuned when irradiated with laser beams of properly chosen photon energy. The hydrophilicity is enhanced upon UV laser irradiation since the embedded nonpolar spiropyran molecules convert to their polar merocyanine isomers. The process is reversed upon green laser irradiation. Structuring of the photochromic polymeric surfaces with soft lithography enhances significantly the hydrophobicity of the system, indicating that the water droplets on the patterned features interact with air that is trapped in the microcavities, thus creating superhydrophobic air-water contact areas. Furthermore, the light-induced wettability variations of the structured surfaces are enhanced by a factor of 3 compared to those on the flat surfaces. This significant enhancement is attributed to the photoinduced reversible volume changes to the imprinted gratings, which additionally contribute to the wettability changes due to the light-induced photochromic interconversions.
\end{abstract}

\section{Introduction}

The understanding and fabrication of functional surfaces with controlled wetting properties, which can respond to external stimuli, ${ }^{1-8}$ has attracted the interest of the scientific community, due to their wide range of potential applications, including microfluidic devices, ${ }^{9}$ controllable drug delivery, ${ }^{10}$ and selfcleaning surfaces. ${ }^{11}$ In this respect, a significant amount of effort has been devoted to surfaces functionalized with photochromic molecules due to their reversible wettability changes upon light irradiation. In particular, Ichimura et al. ${ }^{12}$ and Radüge et al. ${ }^{13}$ have reported wettability changes upon light irradiation of a surface modified with a photoisomerizable monolayer based on

* To whom correspondence should be addressed: E-mail: nassia@ iesl.forth.gr (A.A.); spiros@iesl.forth.gr (S.H.A.).

FORTH.

$\doteqdot$ University of Patras.

$\S$ CNR.

"University of Crete.

(1) Lahann, J.; Mitragotri, S.; Tran, T.-N.; Kaido, H.; Sundaram, J.; Choi, I.

S.; Hoffer, S.; Somorjai, G. A.; Langer, R. Science 2003, 299, 371-374.

(2) Coupe, B.; Evangelista, M. E.; Yeung, R. M.; Chen, W. Langmuir 2001, 17, 1956-1960.

(3) Abbott, N. L.; Gorman, C. B.; Whitesides, G. M. Langmuir 1995, 11, $16-18$

(4) Dupont-Gillain, Ch. C.; Adriaensen, Y.; Derclaye, S.; Rouxhet, P. G. Langmuir 2000, 16, 8194-8200.

(5) Anastasiadis, S. H.; Retsos, H.; Pispas, S.; Hadjichristidis, N.; Neophytides, S. Macromolecules 2003, 36, 1994-1999.

(6) Isaksson, J.; Tengstedt, C.; Fahlman, M.; Robinson, N.; Berggren, M. Adv Mater. 2004, 16, 316-320.

(7) Chiou, P. Y.; Moon, H.; Toshiyoshi, H.; Kim, C.-J.; Wu, M. C. Sens. Actuators, A 2003, 104, 222-228.

(8) Minko, S.; Müller, M.; Motornov, M.; Nitschke, M. J. Am. Chem. Soc.

2003, 125, 3896-3900.

(9) Zhao, B.; Moore, J. S.; Beebe, D. J. Science 2001, 291, 1023-1026.

(10) A La Van, D.; McGuire, T.; Langer, R. Nat. Biotechnol. 2003, 21, $1184-$ 1191

(11) Blossey, R. Nat. Mater. 2003, 2, 301-306.

(12) Ichimura, K.; Oh, S.-K.; Nakagawa, M. Science 2000, 288, 1624-1626.

(13) Radüge, C.; Papastavrou, G.; Kurth, D. G.; Motschmann, H. Eur. Phys.

J. E 2003, 10, 103-114. azobenzene. Rosario et al. ${ }^{14}$ used a photoresponsive monolayer based on spiropyran (SP) to coat capillary tubes, which showed a higher water rise after UV irradiation than after visible irradiation. Möller et al..$^{15}$ used monolayers of a polymeric material containing azobenzene chromophores in its side chains to create reversible patterns of microdroplets using light illumination and a mask.

Recently, substrates incorporating photochromic molecules were examined with emphasis on a totally different aspect. It was shown that a polymeric matrix doped with photochromic SP molecules can alter its macroscopic volume reversibly upon irradiation cycles of UV and green laser pulses. ${ }^{16} \mathrm{In}$ particular, reversible bending of an $\mathrm{SP}$-polymer film was monitored due to mechanical cycles of contraction/lengthening, which are induced and controlled by UV and green laser pulses. In the present work we exploit further the capability of this type of substrate to undergo light-controlled volume changes combined with reversible changes in its polarity and, thus, in its wetting properties in response to the external stimulus of light. We study the impact of surface topology on the wetting of nanopatterned $\mathrm{SP}$-polymer surfaces upon alternating UV-vis laser light irradiation, combining in this way the wettability changes and the dimensional changes of the pattern due to the light-induced photochromic transformations. The light-induced wettability changes of the photochromic polymer system are significantly enhanced by nanopatterning. This finding is related to the fact that the water drops placed on the patterned area are situated partially on air, which is trapped below the drops within the patterned features. The dimensional changes, induced in the patterned features by light irradiation, influence the water-air

(14) Rosario, R.; Gust, D.; Hayes, M.; Jahnke, F.; Springer, J.; Garcia, A Langmuir 2002, 18, 8062-8069.

(15) Möller, G.; Harke, M.; Motschmann, H. Langmuir 1998, 14, 4955-4957.

(16) Athanassiou, A.; Kalyva, M.; Lakiotaki, K.; Georgiou, S.; Fotakis, C. Adv. Mater. 2005, 17, 988-992. 
interface and, thus, strongly affect the extent of the wettability changes of the surface.

\section{Materials and Methods}

Sample Preparation. The light-switchable surfaces used in this work are easily prepared, since no surface chemistry is involved. Poly(ethyl methacrylate)-co-poly(methyl acrylate), P(EMA)-co$\mathrm{P}(\mathrm{MA})$, with an average $M_{\mathrm{w}} \approx 100000$, was purchased from Aldrich and used as received. Its glass transition temperature, $T_{\mathrm{g}}$, was $48^{\circ} \mathrm{C}$. The photochromic molecule that was used was $1^{\prime}, 3^{\prime}$-dihydro- $1^{\prime}, 3^{\prime}, 3^{\prime}$ trimethyl-6-nitrospiro[2H-1-benzopyran-2, $2^{\prime}-(2 H)$-indole] $\left(6-\mathrm{NO}_{2}-\right.$ BIPS), which belongs to the family of spiropyrans. The flat films were prepared by spin coating toluene solutions of 95/5 mixtures of $\mathrm{P}(\mathrm{EMA})$-co-P(MA) and spiropyran on glass substrates to produce homogeneous films.

Nanopatterning by soft lithography was used for the preparation of nanoimprinted films. The patterning of the surface of the samples is done using soft molding, ${ }^{17}$ which has been previously demonstrated to be a powerful approach to pattern functional organic materials. ${ }^{18}$ More specifically, the master structures were fabricated (typically gratings with a period of about $1.3 \mu \mathrm{m}$ ) onto quartz by either photolithography or electron beam lithography, and subsequent reactive ion etching. Next, their elastomeric replicas were realized by poly(dimethylsiloxane) (Dow Corning Sylgard 184) according to a standard replica molding procedure and placed onto the flat spin-coated photochromic polymer films under their own weight. The system was then heated to $55^{\circ} \mathrm{C}$, namely, a temperature higher than the glass transition temperature of the P(EMA)-co-P(MA) films. After the thermal cycle, the replica was easily peeled from the SPpolymer substrate, onto which the pattern was faithfully transferred. The soft molding was carried out in a nitrogen atmosphere to avoid the deterioration of the photochromic molecules upon heating. It is based on the capillarity effect that drives a polymer, or an appropriate chosen blend of organics, to penetrate into the recessed features of an elastomeric element previously templated by a suitable master. A low polymer viscosity favors such a penetration. The proper increase of temperature reduces the viscosity of the organic compound, because of the increased molecular mobility, thus allowing the filling of the pattern in a few minutes.

Experimental Methods. The sessile drop geometry was selected for determining the contact angle of water on the solid SP-P(EMA)co-P(MA) and $\mathrm{P}$ (EMA)-co-P(MA) substrates. The method is based on the principle that the profile of a sessile drop of a fluid on a solid surface is governed by a force balance between the surface/interfacial tension and gravity. The contact angle of water was measured in air using an automated tensiometer, which utilizes the whole drop profile for the evaluation of both the contact angle and the surface tension of the fluid. The advantage of this method for the determination of the contact angles is due to the fact that use is made of the whole drop profile and not of just the contact points with the substrate surface; the actual value of the contact angle is then extracted from the data and is not subjected to the influence of possible impurities right at the drop edges at the surface. A Material Interface Associates, Inc. automated tensiometer was used to determine the contact angles, which utilizes a collection of digital images of sessile drops. Each drop ( $3 \mu \mathrm{L})$ of distilled, diionized Millipore water (18.2 M $\Omega$ ) was formed from a capillary tip, and was detached gently from the tip upon the substrate of interest. The atmosphere around the drop was rich in water vapor to achieve minimum evaporation of the droplet. The contact angle was obtained upon equilibration of the drop. Before irradiation the sample is dried, and after irradiation a new drop is used for the measurement.

The reversible wetting properties of the samples are examined upon UV and visible pulsed laser irradiation. The lasers used for the irradiation of the samples were a $\mathrm{XeCl}$ laser operating at $308 \mathrm{~nm}$, $\tau_{\text {pulse }} \approx 30$ ns (Lambda Physik, EMG $201 \mathrm{MSC}$ ), and a Nd:YAG laser, operating at the second harmonic, $\lambda=532 \mathrm{~nm}, \tau_{\text {pulse }} \approx 5 \mathrm{~ns}$

(17) Suh, K. Y.; Kim, Y. S.; Lee, H. H. Adv. Mater. 2001, 13, 1386-1389. (18) Pisignano, D.; Persano, L.; Gigli, G.; Cingolani, R.; Babudri, F.; Farinola, G. M.; Naso, F. Appl. Phys. Lett. 2004, 84, 1365-1367.
(B. M. Industries, series 5000). The laser beams were focused weakly onto the surface of the samples overlapping at an area of $3.5 \times 3.5$ $\mathrm{mm}^{2}$. After the measurement of the initial contact angle, the drop was removed and the flat or patterned sample was irradiated with $50 \mathrm{UV}$ pulses of fluence $F_{\text {laser }}=40 \mathrm{~mJ} \mathrm{~cm}^{-2}$. The new contact angle of a water drop was measured after the UV irradiation. The sample was then again dried and irradiated with 200 green laser pulses of fluence $F_{\text {laser }}=45 \mathrm{~mJ} \mathrm{~cm}^{-2}$. The new contact angle of a water drop was measured after the green irradiation.

\section{Results and Discussion}

The apparent contact angle of a drop on a flat substrate, $\theta_{\mathrm{Y}}$, formed when the liquid is in contact with a solid surface in static equilibrium with its vapor, is determined by Young's equation

$$
\gamma_{\mathrm{LV}} \cos \theta_{\mathrm{Y}}=\gamma_{\mathrm{SV}}-\gamma_{\mathrm{SL}}
$$

where $\gamma_{\mathrm{LV}}, \gamma_{\mathrm{SV}}$, and $\gamma_{\mathrm{SL}}$ represent the interfacial tensions at the boundaries between the liquid (L), vapor (V), and solid (S).

Figure 1 shows characteristic images of water drops, which lie on the SP-doped polymeric substrate before irradiation, after irradiation with 50 UV laser pulses (second cycle), and after subsequent irradiation with 200 green pulses (second cycle). These are shown together with the average values of the contact angles of drops lying on the initial, the UV-irradiated, and the green-irradiated flat photochromic polymeric surfaces. The values are taken from drops on a number of different samples of the same composition. The contact angle values after the UV and green irradiation are limited to the first two UV - green irradiation cycles to ensure limitation of photochemical phenomena, which cause degradation of the photochromic molecules. The aging and degradation of the system upon multiple irradiation cycles will be the subject of a forthcoming study.

The maximum difference between the average contact angles measured in the experiment on the flat surfaces shown in Figure 1 is $7^{\circ} \pm 1^{\circ}$. When the SP-doped PEMMA sample is irradiated with UV pulses, the SP molecules convert to their merocyanine (MC) isomers and the surface becomes colored. Since the MC stereoisomers exhibit an enhanced dipolar moment compared to that of the SP isomers, ${ }^{19}$ the surface of the sample becomes more hydrophilic and, thus, the contact angles of the water drops decrease. Subsequent irradiation of the sample with green laser pulses converts the molecules back to the initial SP form, and thus, the surface becomes more hydrophobic, resulting again in an increase of the contact angles. The number and the energy density of the UV pulses used in the experiment presented in Figure 1 ensure complete conversion of the SP molecules to their MC isomeric form, according to spectroscopic studies. The number and the energy density of the green pulses ensure maximum recovery of the contact angles of the water drops. Additional green laser pulses do not influence the wettability of the surfaces any further. For comparison reasons, the average values of the contact angles of water drops on a flat polymer surface without SP molecules are also presented in Figure 1. It is obvious that laser irradiation does not have any impact on the contact angles since the light-induced interconversions of the doped photochromic molecules are exclusively responsible for the changes in the wetting properties.

Extensive investigations, which have been performed on roughened solid surfaces, clearly show that the roughness can strongly influence the contact angle of a liquid drop on them. ${ }^{20-23}$

(19) Abe, Y.; Nakao, R.; Horii, T.; Okada, S.; Irie, M. J. Photochem. Photobiol., A 1996, 95, 209-214.

(20) Yoshimitsu, Z.; Nakajima, A.; Watanabe, T.; Hashimoto, K. Langmuir 2002, 18, 5818-5822. 
(a)
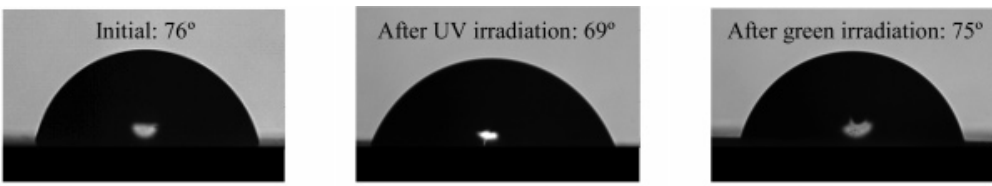

(b)

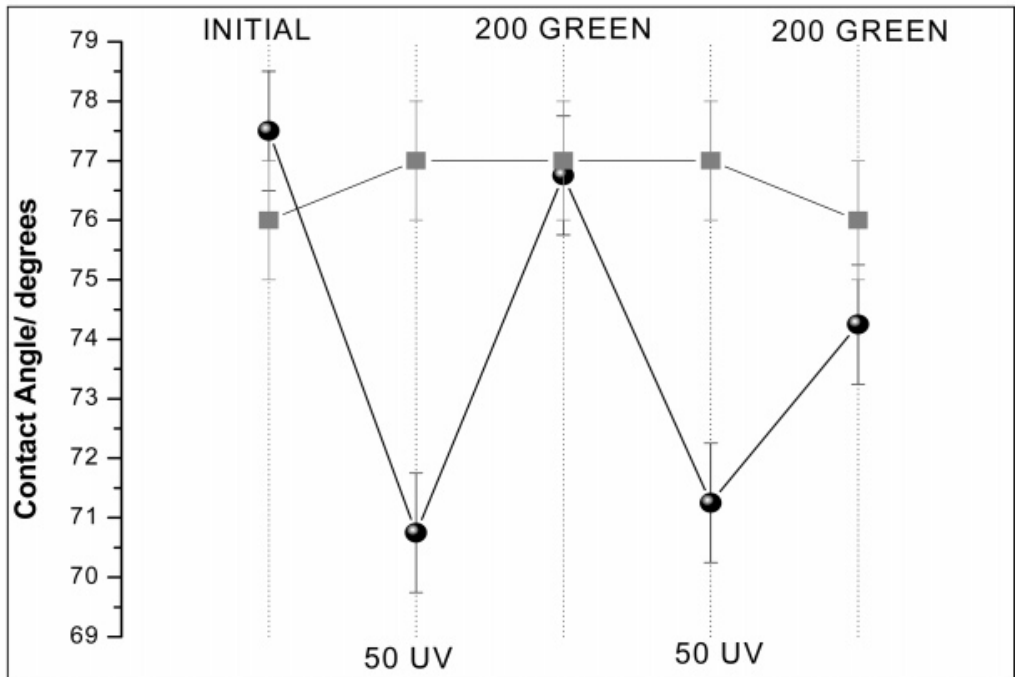

Figure 1. (a) Characteristic images of water drops residing on the initial, the UV-irradiated, and the green-irradiated flat surfaces of 5\% SP-95\% P(EMA)-co-P(MA) samples. (b) Average contact angle values of water drops residing on the initial, the UV-irradiated, and the green-irradiated flat surfaces of 5\% SP-95\% P(EMA)-co-P(MA) samples (-) and average contact angle values of water drops situated on the initial, the UV-irradiated, and the green-irradiated flat surface of a P(EMA)-co-P(MA) sample without photochromic molecules ( $\mathbf{\square})$. The two first irradiation cycles are presented, each irradiation cycle consisting of $50 \mathrm{UV}$ laser pulses of fluence $40 \mathrm{~mJ} \mathrm{~cm}-2$ and 200 green laser pulses of fluence $45 \mathrm{~mJ} \mathrm{~cm} \mathrm{~cm}^{-2}$.

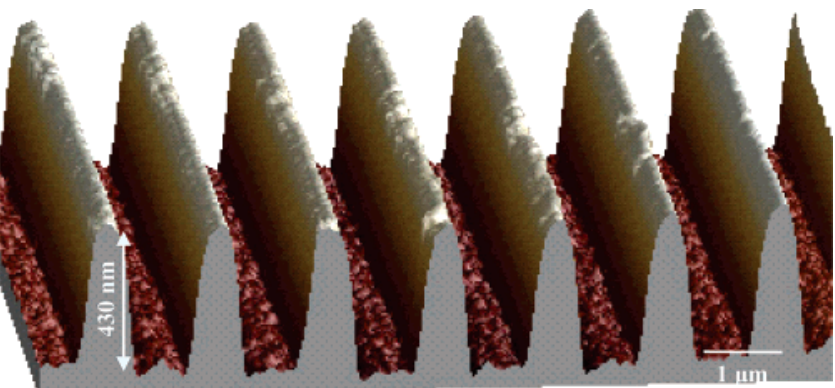

Figure 2. Atomic force microscopy image of the nanoimprinted grating formed on the surface of the $5 \% \mathrm{SP}-95 \% \mathrm{P}(\mathrm{EMA})$-co$\mathrm{P}(\mathrm{MA})$ sample. The $x$ and $y$ axes are presented on different scales.

To determine whether the wetting of the SP-doped polymeric surfaces and its light-induced reversibility can be further affected by the surface topology, we examined next these surfaces patterned with gratings using soft lithography. The work is done with fixed topological parameters, such as periodicity and duty cycle, for the understanding of the basic issues. The imprinted grating formed on the surface of a sample is shown in Figure 2 .

Indeed, patterning of the polymer surface greatly affects its wettability. The values of the water contact angles on the patterned surfaces are always greater than on the flat surfaces, and they vary from $\theta_{\mathrm{r}} \approx 95^{\circ}$ to $\theta_{\mathrm{r}} \approx 113^{\circ}$ depending on some structural variations on the imprinted gratings, explored in detail in the following discussion. Figure 3 shows the Millipore water contact angle values on the patterned surfaces for the two first UVgreen irradiation cycles, together with characteristic images of water drops situated on a surface before irradiation, after UV

(21) Bico, J.; Marzolin, C.; Quéré, D. Europhys. Lett. 1999, 47, 220-226.

(22) Patankar, N. A. Langmuir 2003, 19, 1249-1253.

(23) Lafuma, A.; Quéré, D. Nat. Mater. 2003, 2, 457-460. irradiation (second cycle), and after subsequent green irradiation (second cycle). The two presented cases are the average contact angle values from different samples of the same composition having initial contact angles of $>105^{\circ}$ and $\leq 105^{\circ}$. The maximum average contact angle difference measured in the experiments shown in Figure 3 is $19^{\circ} \pm 3^{\circ}$. It is obvious, comparing these results with the ones presented in Figure 1 for the flat surfaces, that the nanostructuring enhances significantly the difference between the contact angles of the water drops formed on the initial, the UV-irradiated, and the green-irradiated surfaces. Specifically, the light-induced contact angle changes on the smooth surfaces due to the photochromic transformations are further enhanced by a factor of almost 3 on the patterned surfaces. As Rosario et al. ${ }^{24}$ have demonstrated, the light-induced contact angle changes can be amplified by a factor of nearly 2 even in the case of randomly roughened surfaces, covered by a hydrophobic monolayer of SP molecules, relative to smooth surfaces. They used fractally rough $\mathrm{Si}$ nanowires covalently bound with the photochromic molecules, grown on a Si substrate. For comparison reasons, the contact angle average values of water drops on a nanoimprinted polymer surface without SP molecules are also presented in Figure 3. As expected, since photochromic molecules are not incorporated into the polymer matrix, laser irradiation does not influence the contact angle.

Two distinct hypotheses, which are graphically presented in Figure 4, are classically proposed to explain the effect of roughness on the wetting of a surface. The first is referred to as the Wenzel model, and it proposes that roughness increases the liquid-solid interfacial area, and thus, hydrophilicity or hydrophobicity is enhanced geometrically. ${ }^{25}$ In particular, hydrophilic surfaces $(\theta$ $\left.<90^{\circ}\right)$ become more hydrophilic, and hydrophobic surfaces $(\theta$

(24) Rosario, R.; Gust, D.; Garcia, A. A.; Hayes, M.; Taraci, J. L. J. Phys Chem. B 2004, 108, 12640-1264.

(25) Wenzel, R. N. Ind. Eng. Chem. 1936, 28, 988-994. 
(a)
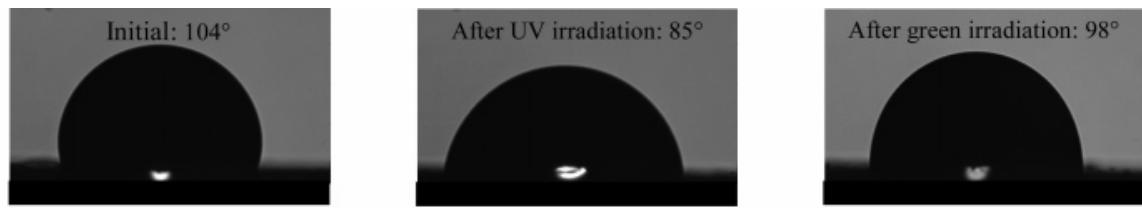

(b)

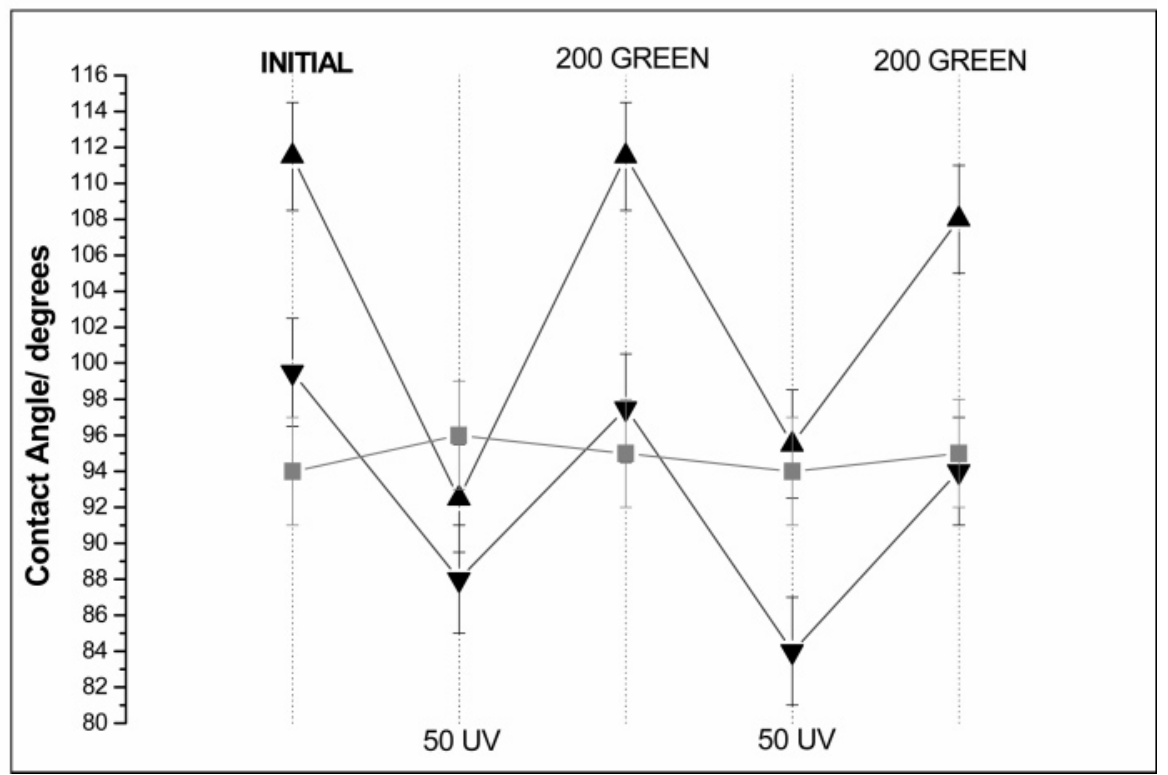

Figure 3. (a) Characteristic photographs of water drops situated on the initial, the UV-irradiated, and the green-irradiated nanoimprinted surface of a 5\% SP-95\% P(EMA)-co-P(MA) sample having an initial contact angle of $104^{\circ}$. (b) Average contact angle values of water drops situated on the initial, the UV-irradiated, and the green-irradiated nanoimprinted surface of different 5\% SP-95\% P(EMA)-co-P(MA) samples having an initial contact angle of $>105^{\circ}(\mathbf{\Lambda})$, average contact angle values of water drops situated on the initial, the UV-irradiated, and the green-irradiated nanoimprinted surface of different $5 \%$ SP-95\% P(EMA)-co-P(MA) samples having an initial contact angle of $\leq 105^{\circ}(\boldsymbol{\nabla})$, and average contact angle values of water drops situated on the initial, the UV-irradiated, and the green-irradiated nanoimprinted surface of a P(EMA)-co-P(MA) sample without photochromic molecules (ם). The two first irradiation cycles are presented, each cycle consisting of $50 \mathrm{UV}$ laser pulses of fluence $40 \mathrm{~mJ} \mathrm{~cm}{ }^{-2}$ and 200 green laser pulses of fluence $45 \mathrm{~mJ} \mathrm{~cm}^{-2}$.
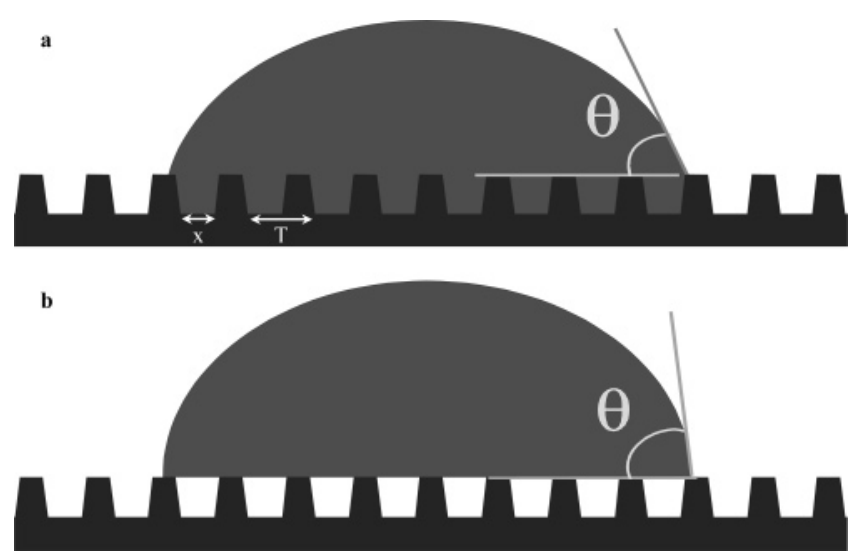

Figure 4. (a) Liquid drop residing on the patterned surface according to the Wenzel model. The liquid fills the capillaries of the imprinted grating. (b) Liquid drop residing on the patterned surface according to the Cassie-Baxter model. Air is trapped below the drop in the capillaries of the imprinted grating.

$>90^{\circ}$ ) become more hydrophobic. The second hypothesis belongs to Cassie and Baxter, and it proposes that air can remain trapped below the drops. ${ }^{26}$ Since the drop is situated partially on air, the surface exhibits an enhanced hydrophobic behavior.

In both models, the apparent contact angle of a drop on a rough substrate, $\theta_{\mathrm{r}}$, is related to Young's contact angle $\theta_{\mathrm{Y}}$, determined on a flat surface of the same nature. In the Wenzel model, the relation between the two contact angles is

(26) Cassie, A. B. D.; Baxter, S. Trans. Faraday Soc. 1944, 40, 546-551.

$$
\cos \theta_{\mathrm{r}}=r \cos \theta_{\mathrm{Y}}
$$

where the surface roughness $r$ is defined as the ratio of the actual wetted surface area over the geometric surface area of the substrate. The latter is the surface as measured on the plane of the interface. ( $r$ is a number larger than unity which equals unity for flat surfaces.)

In the Cassie-Baxter model, the contact angle of the drop, $\theta_{\mathrm{r}}$, is an average between the value on air $\left(180^{\circ}\right)$ and the value on the flat surface $\left(\theta_{\mathrm{Y}}\right)$, and is given by

$$
\cos \theta_{\mathrm{r}}=-1+f_{\mathrm{s}}\left(1+\cos \theta_{\mathrm{Y}}\right)
$$

where $f_{\mathrm{s}}$ is the solid fraction of the surface in contact with the liquid $\left(f_{\mathrm{s}}\right.$ is dimensionless and smaller than unity).

According to Wenzel's model, the wetting of the roughened surface examined herein would be better compared to that of the flat surface $\left(\theta_{\mathrm{r}}<\theta_{\mathrm{Y}}\right)$, since the initial contact angles for the flat surface $\left(\theta_{\mathrm{Y}} \approx 77.5\right.$; , see Figure 1$)$ imply a slightly hydrophilic situation $\left(\theta_{Y}<\pi / 2\right)$. On the contrary, the measured contact angles on the patterned surfaces are always greater than that on the flat surface $\left(\theta_{\mathrm{r}}=95^{\circ}-113^{\circ}\right)$. Therefore, the Wenzel model is not valid for the present situation, and one can safely assume that the wetting of the imprinted surface follows the CassieBaxter model. Given that the average initial contact angle on the flat surface is $\theta_{\mathrm{Y}} \approx 77.5^{\circ}$ (Figure 1) and the initial contact angle on the patterned surface, $\theta_{\mathrm{r}}$, varies from $95^{\circ}$ to $113^{\circ}$, it is calculated from eq 3 that the factor $f_{\mathrm{s}}$ can take values in the range $0.50-$ 0.75 . The lower value 0.50 is achieved when the pattern is fully symmetric with a top hat profile, and the water drop is lying on 
the top of each stripe. In this case the factor $f_{\mathrm{s}}$ can be calculated as $f_{\mathrm{s}}=(T-x) / T=0.50$, where $T$ is the period of the grating and $x=T / 2$ is the width of each channel (see Figure 4). Since this value is the lower limit calculated according to the experimental contact angles, it is assumed that the water droplets do not always lie on the top of the structure but they can sink down into the channels, resulting in a higher surface fraction in contact with the liquid (higher $f_{\mathrm{s}}$ values) and consequently smaller contact angles. This can be attributed to the fact that the stripes do not always have perfect top hat profiles, as demonstrated in Figure 2. Therefore, the variations of the initial contact angles on the patterned surfaces depend on the slope variations of the sidewalls of the channels, since they can affect the area of the surface that is wetted by the water drop, which determines the factor $f_{\mathrm{s}}$ in eq 3 . The role of the topological characteristic of the pattern, such as periodicity and duty cycle, on the wetting will be systematically addressed in a separate study.

The enhancement in the contact angle differences can be attributed to the light-induced structural changes on the nanoimprinted grating. Indeed, AFM measurements on the gratings show that the full width at half-maximum of the stripes decreases following UV irradiation, whereas it increases back to the initial value following green irradiation. The average value of contraction of the nanoimprinted stripes is translated into a $30 \mathrm{~nm}$ decrease of the full width at half-maximum. This average value is extracted from patterns on three different samples. The structural changes that occur in the pattern following the UV irradiation seem to decrease the contact angle, contributing further to the decrease due to the photochromic transformation of SP to MC. According to eq 3 , the contact angle of the water drop decreases when the fraction of the solid, which is in contact with the liquid $\left(f_{\mathrm{s}}\right)$, increases. This implies that as the stripes of the pattern are narrower the water drop penetrates deeper into the channels of the UV-irradiated pattern. Therefore, it can be assumed that the Cassie-Baxter model of air trapping is still valid after UV irradiation of the pattern but the liquid-solid interfacial area increases. After irradiation with green laser pulses, the MC molecules return to their SP isomers, the stripes recover their previous volume, and thus the drop returns to its previous condition, demonstrating once more that the wetting behavior is greatly influenced by both the changes in the surface polarity and the conformational changes.

\section{Conclusions}

In this work, it is demonstrated how surface chemistry and structure can be combined to influence the wetting behavior of polymeric surfaces. In particular, the photoinduced reversible wettability changes of a spiropyran-containing polymer film surface can be greatly enhanced (by as much as a factor of 3 ) and, in principle, controlled by micropatterning the surface with soft molding lithography. The technique is well-established for surface structuring, and thus, the patterned surfaces as well as the reversible wetting behavior upon specific laser irradiation are fully reproducible.

Acknowledgment. We thank Mrs. A. Manousaki (IESLFORTH) for her valuable help in the atomic force microscopy measurements. Part of this research was sponsored by NATO's Scientific Affairs Division (Science for Peace Programme) and by the Greek General Secretariat of Research and Technology (PENED Programme 01ED587).

LA052122G 